\title{
Исследование напряженности внутренних электрических полей в активной области светодиодных структур на основе InGaN/GaN с разным числом квантовых ям методом спектроскопии электропропускания
}

\author{
() А.Э. Асланян ${ }^{1}$, Л.П. Авакянц ${ }^{1}$, А.В. Червяков ${ }^{1}$, А.Н. Туркин ${ }^{1}$, С.С. Мирзаи ${ }^{1}$, \\ В.А. Курешов ${ }^{2}$, Д.Р. Сабитов ${ }^{2}$, А.А. Мармалюк $^{2}$ \\ ${ }^{1}$ Московский государственный университет им. М.В. Ломоносова (физический фракультет), \\ 119991 Москва, Россия \\ ${ }^{2} \mathrm{OOO} \mathrm{„Сигм} \mathrm{плюс“,}$ \\ 117342 Москва, Россия \\ E-mail: aslanyan.artyom@physics.msu.ru
}

Поступила в Редакцию 16 декабря 2019 г.

В окончательной редакции 24 декбаря 2019 г.

Принята к публикации 24 декабря 2019 г.

\begin{abstract}
Методом спектроскопии электропропускания исследованы внутренние электрические поля светодиодных гетероструктур зеленого свечения на основе InGaN/GaN с разным числом квантовых ям в активной области. Проведено отнесение частот наблюдаемых спектральных линий с возможными типами межзонных переходов. Обнаружено увеличение числа межзонных переходов типа „квантовая яма-квантовый барьер“ по мере увеличения числа квантовых ям, что объясняется неодинаковой степенью сегрегации атомов In в разные барьерные слои $\mathrm{GaN}$. C помощью серии спектров электропропускания при разном смещении $p-n$-перехода рассчитана напряженность внутренних электрических полей в квантовых ямах. Обнаружено, что с ростом числа квантовых ям напряженность внутреннего пьезоэлектрического поля уменьшается с 3.20 до $2.82 \mathrm{MB} / \mathrm{cм}$.
\end{abstract}

Ключевые слова: светодиодные гетероструктуры, модуляционная спектроскопия, квантовая яма, нитрид галлия.

DOI: $10.21883 /$ FTP.2020.04.49151.9335

\section{1. Введение}

Как установлено ранее $[1,2]$, внутренние электрические поля, возникающие вследствие пьезоэлектрического эффекта в квантовых ямах (КЯ) активной области светодиодных гетероструктур $\mathrm{GaN} / \mathrm{InGaN} / \mathrm{GaN}$ отрицательно влияют на эффективность свечения. Особенно сильно это проявляется при росте структуры на сапфировой подложке в полярном направлении вдоль оси c $\mathrm{GaN}$ [3]. Возникающие в этом случае пьезоэлектрические поля приводят к изменению зонной структуры и смещению волновых функций электронов и дырок к противоположным гетерограницам в каждой КЯ. Соответствующее уменьшение интеграла перекрытия приводит к уменьшению вероятности рекомбинации носителей в активной области [4]. Рассогласование постоянных кристаллической решетки на границах $\mathrm{InGaN} / \mathrm{GaN}$ также приводит к возникновению пьезоэлектрических полей. Такие рассогласования минимизируют с помощью подбора толщин слоев гетероструктуры InGaN/GaN, a также с помощью добавления буферных сверхрешеток InGaN/GaN с малым содержанием индия в активную область [5-7]. Согласно работе [8], излучательная рекомбинация может происходить не во всех КЯ активной области. Кроме того, ряд работ указывает на наличие неоднородного распределения напряженности внутрен- них электрических полей по толщине активной области $[9,10]$. Таким образом, исследования распределения напряженности пьезоэлектрических полей по толщине активной области могут способствовать оптимизации дизайна активной области с целью повышения излучательной способности светодиодов на основе гетероструктур $\mathrm{InGaN} / \mathrm{GaN}$.

Обычно для оценки напряженности электрического поля в активной области исследуют зависимость энергии перехода между основными (невозбужденными) уровнями в КЯ $E_{1 e 1 h h}$ валентной зоны и зоны проводимости от внешнего электрического поля $F_{\text {ext }}[11]$. В основе такого подхода лежит квантово-размерный эффект Штарка (QCSE). Оценка напряженности электрического поля может быть получена с помощью исследования механизма компенсации внешним электрическим полем внутреннего. В этом случае модуль напряженности внутреннего электрического поля сравнивается с напряженностью внешнего при максимальной энергии межзонного перехода. Также можно аппроксимировать зависимость $E_{1 e 1 h h}\left(F_{\text {ext }}\right)$, где напряженность внутреннего электрического поля является параметром [12].

Существует ряд спектральных методов, применяемых для получения указанных энергий переходов $E_{1 e 1 h h}$. Метод электролюминесценции [13] трудно использовать для оценки пьезоэлектрических полей, посколь- 
ку при свечении светодиодной структуры возникает эффект экранировки внутреннего электрического поля носителями заряда в КЯ [1]. Энергии переходов, полученные из широких спектральных линий фотолюминесценции, зачастую трудно точно определить изза возникающих интерференционных эффектов и слабого сигнала при больших напряженностях внешних компенсирующих электрических полей [11]. Использование метода электроотражения также сопряжено с неоднозначностью определения энергии перехода изза несимметричности спектральных линий, связанных с осцилляциями Франца-Келдыша [14]. При использовании соотношений Крамерса-Кронига в этом случае могут возникать ложные пики, не связанные со структурой [15].

Метод электропропускания, используемый в данной работе, с одной стороны, обладает преимуществом люминесцентных спектральных методов, так как спектральные линии оказываются симметричными, что упрощает определение энергий соответствующих переходов. Это особенно важно при наличии переходов с близкими значениями соответствующих энергий, когда пики плохо разрешены. С другой стороны, данный метод имеет преимущества модуляционных методик (например, электроотражения), позволяя наблюдать узкие спектральные линии с высоким соотношением сигнал/шум даже при комнатной температуре. Несмотря на указанные достоинства, метод электропропускания использовался крайне редко для структур с КЯ $\mathrm{InGaN} / \mathrm{GaN}$, в отличие от выше перечисленных методов. По нашим данным опубликовано всего одна работа, где используется метод электропропускания [16], а также несколько работ, где применялись похожие методы электропоглощения $[13,17,18]$ и фотопропускания [19].

В настоящей работе методом спектроскопии электропропускания исследуются напряженности внутренних электрических полей в активной области светодиодных структур на основе InGaN/GaN с разным числом квантовых ям в активной области $p-n$-перехода.

\section{2. Образцы. Методика регистрации спектров ЭП}

Исследуемые образцы светодиодных гетероструктур на основе InGaN/GaN с разным числом КЯ в активной области были выращены методом газофазной эпитаксии из металлоорганических соединений (MOCVD) на сапфировых подложках толщиной 0.43 мм и диаметром $50.8 \mathrm{MM}$.

На рис. 1 показана схема расположения слоев гетероструктуры. На подложке последовательно выращены: затравочный низкотемпературный и буферные слои $i$-GaN (2 мкм), n-GaN (3 мкм), легированный $\mathrm{Si}$ с концентрацией доноров $N_{D}=5 \cdot 10^{18} \mathrm{~cm}^{-3}$, затем первый барьерный слой активной области $\mathrm{GaN}(16 \mathrm{HM})$ и последующие квантовые ямы $\operatorname{In}_{x} \mathrm{Ga}_{1-x} / \mathrm{GaN}(2.5 / 15 \mathrm{Hм})$. В работе

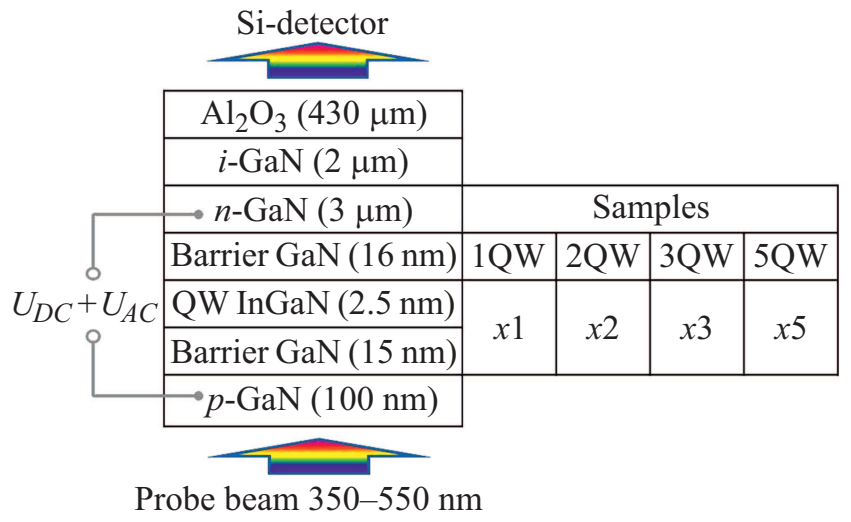

Рис. 1. Структура исследованных образцов с разным количеством КЯ (QW) и схема приложения напряжения на $p-n$-переход. Стрелками показан ход зондирующего луча в методе ЭП.

было исследовано 4 образца с разным количеством КЯ: $1(x=0.24), 2,3$ и $5(x=0.26)$. Последним был выращен слой $p-\mathrm{GaN}$ (110 нм), легированный $\mathrm{Mg}$ с концентрацией акцепторов $N_{A}=2 \cdot 10^{19} \mathrm{~cm}^{-3}$. Контакт от $n$-области был выведен на лицевую поверхность образца. Напряжение к $n$ - и $p$-областям структуры подавалось при помощи прижимных металлических пластин. Одна из пластин имела отверстие, через которое проходило зондирующее излучение. Для минимизации рассеяния света на границах структуры использовались подложки с двухсторонней полировкой.

В эксперименте по ЭП излучение лампы накаливания с помощью монохроматора и системы зеркал фокусировалось на образец под углом $8^{\circ}$, проходило через отверстие в пластине, многослойную структуру и попадало на кремниевый фотоприемник. Изменение коэффициента пропускания под действием модулирующего напряжения регистрировалось методом синхронного детектирования. C генератора на контакты $p-n$-перехода прикладывалось напряжение, содержащее переменную составляющую - меандр со скважностью 2, частотой 370 Гц и амплитудой $U_{A C}=0.125 \mathrm{~B}$ и постоянную составляющую напряжения $U_{D C}-$ смещение $p-n$-перехода (рис. 1). Спектры регистрировались при разных смещениях с шагом 1 В от -20 В до максимального положительного напряжения, при котором отсутствовала излучательная рекомбинация.

\section{3. Результаты и обсуждение}

На рис. 2 приведены спектры ЭП, соответствующие трем смещениям $U_{D C}$. Для определения положения линии, соответствующей фундаментальному переходу в слое InGaN $E_{1 e 1 h h}$, были измерены спектры электроотражения (ЭО) при тех же смещениях (рис. 2). Известно, что спектральный пик расположен между локальными минимумом и максимумом отдельной линии в спектре 


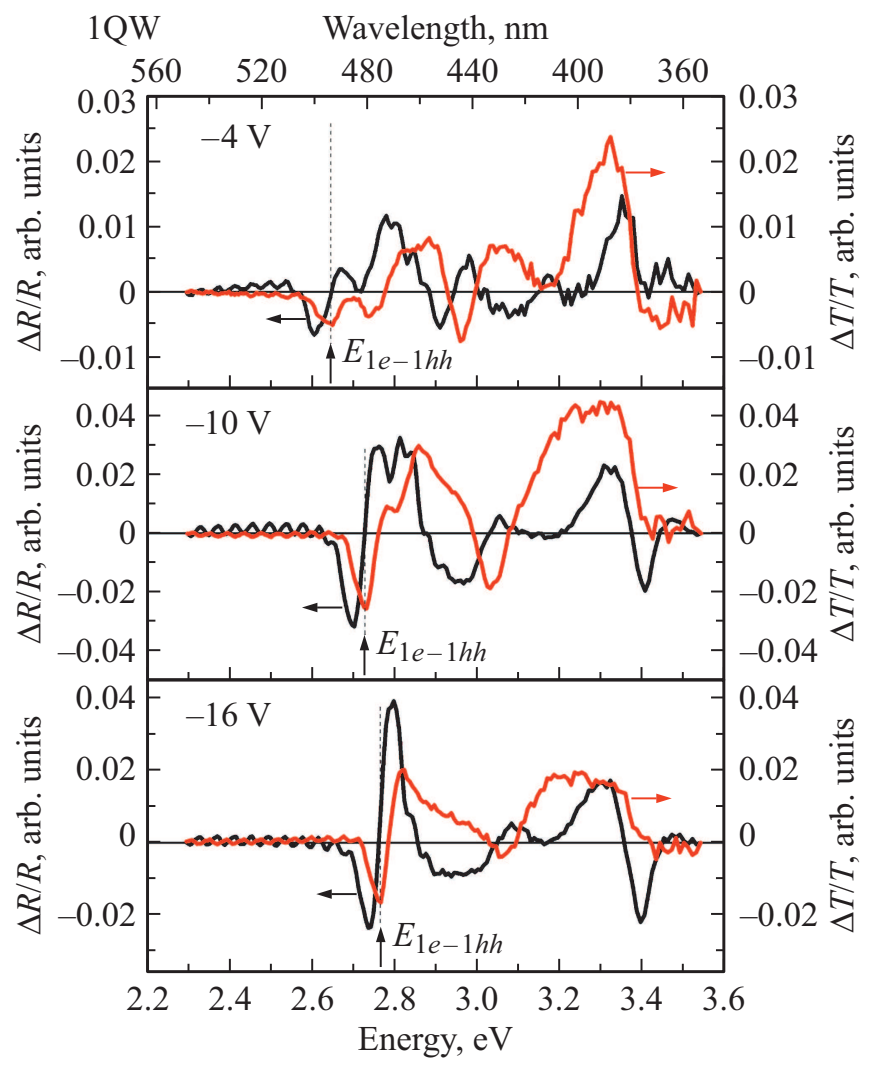

Рис. 2. Спектры ЭО $(\Delta R / R)$ и ЭП $(\Delta T / T)$ для светодиодной гетероструктуры InGaN/GaN с одиночной КЯ при смещениях $U_{D C}=-4,-10$ и -16 В. Стрелками показаны переходы между невозбужденными уровнями в КЯ $E_{1 e 1 h h}$.

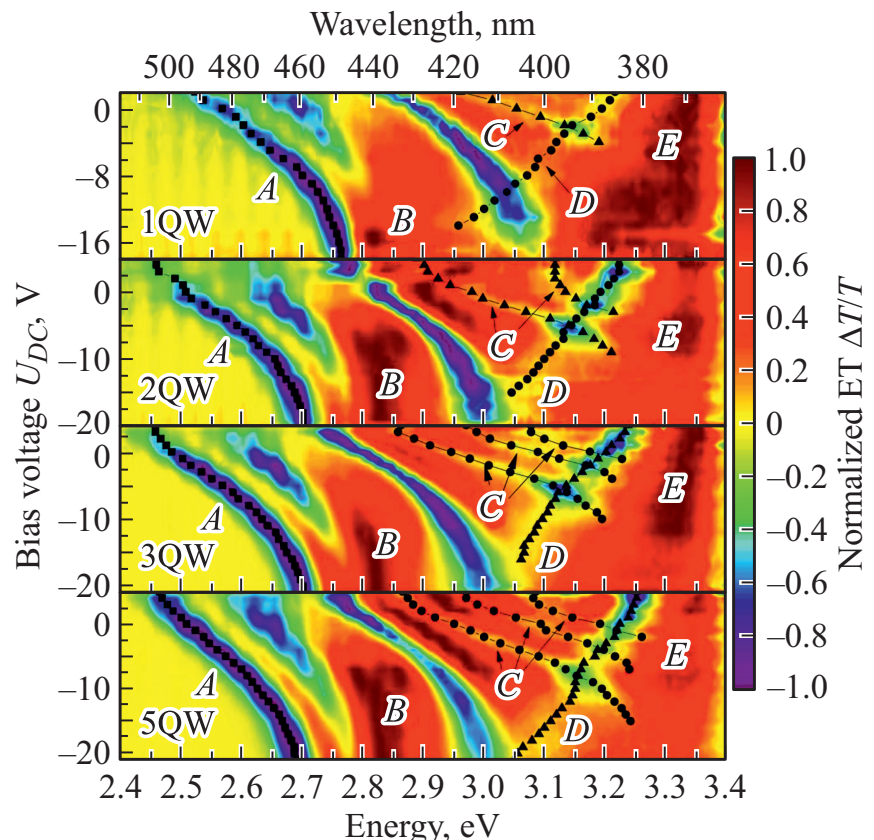

Рис. 3. Нормированные спектры ЭП (ЕТ) при разных смещениях $p-n$-перехода для образцов с разным количеством КЯ $(\mathrm{QW})$ в активной области. Буквами $A-E$ обозначены различные типы переходов, обусловленные зонной структурой (рис. 4). Черными квадратами (ロ) обозначены положения пиков спектральных линий ЭП типа $A$, треугольниками $(\mathbf{\Delta})-$ типа $C$, кружками $(\bullet)-$ типа $D$.
ЭО [20-22]. Видно, что низкоэнергетичный минимум ЭП соответствует указанному положению линии ЭО. Эта информация была использована далее не только для определения фундаментального перехода в слое InGaN, но и для определения других переходов.

Полученные спектры ЭП для всех образцов при разных смещениях $p-n$-перехода были нормированы на максимальное значение сигнала ЭП для более наглядной визуализации сдвигов спектральных линий, связанных с различными межзонными переходами в активной области (рис. 3). Можно выделить следующие пять типов таких межзонных переходов в активной области в порядке возрастания соответствующих энергий (рис. 4).

$A$. Переходы в слоях InGaN между невозбужденными дырочными уровнями КЯ валентной зоны и невозбужденными электронными уровнями КЯ в зоны проводимости. На ненормированных спектрах ЭП эта линия имеет наибольшую интенсивность. Соответствующие длины волн согласуются с данными по длине волны свечения, полученными методом спектроскопии электролюминесценции (ЭЛ) (рис. 5).

$B$. Переходы между возбужденными уровнями в слоях InGaN активной области. Соответствующие энергии согласуются с расчетами уровней энергий для квантовой ямы конечной глубины в отсутствие электрического поля при смещении $U_{D C} \approx-20 \mathrm{~B}$. При таких напряжениях достигается состояние плоских зон КЯ: напряженность внешнего электрического поля компенсирует напряженность внутреннего.

C. Переходы в области гетерограниц InGaN/GaN, размытых вследствие встраивания атомов индия из КЯ InGaN в кристаллическую решетку барьерных слоев $\mathrm{GaN}$. Энергии таких межзонных переходов эквивалентны разностям потенциалов между невозбужденными уровнями в КЯ и барьерными состояниями дырок в валентной зоне или электронов в зоне проводимости слоев $\mathrm{GaN}$, смежных с КЯ. На спектрах ЭП можно наблюдать рост количества переходов типа „С“ с различными энергиями при увеличении количества КЯ. Это объясняется неодинаковым размытием гетерограниц при изготовлении структуры с несколькими КЯ. В этом случае энергии соответствующих переходов будут разными.

$D$. Межзонные переходы в барьерах $\mathrm{GaN}$, заключенных между квантовыми ямами InGaN. Поскольку вектор напряженности пьезоэлектрического поля в барьер $\mathrm{GaN}$ сонаправлен с вектором напряженности внешнего электрического поля, то с ростом обратного напряжения увеличивается суммарная напряженность электрического поля в барьере. Это приводит к красному смещению соответствующей линии.

$E$. Высокоэнергетичная линия 3.35 эВ связана с переходами в слоях $p$-GaN или $n-\mathrm{GaN}$. Несмотря на слабое влияние внешнего электрического поля из-за низкого сопротивления этих слоев, небольшое внутреннее электрическое поле присутствует [23]. Это выражается в небольшом отличии соответствующей энергии в 
(A)
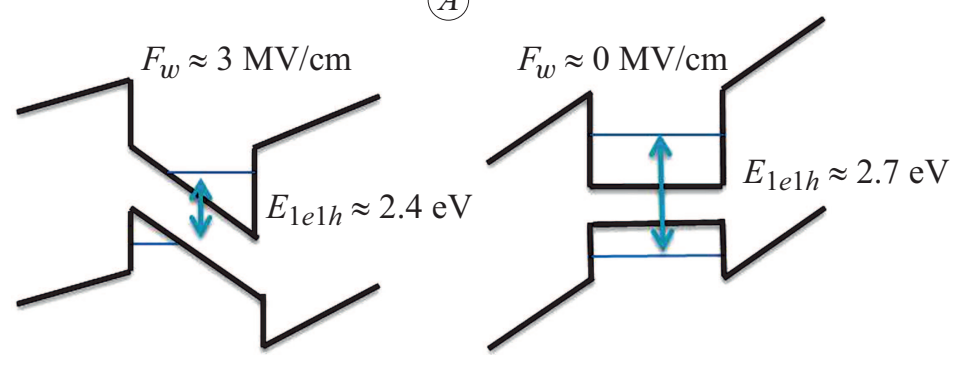

(B)

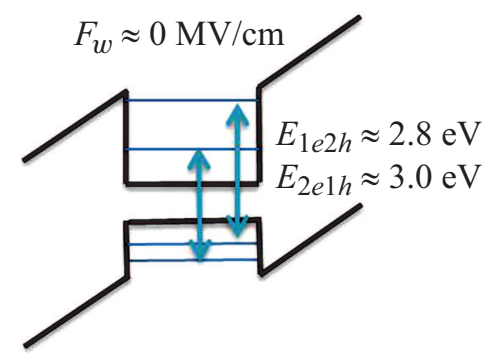

(C)
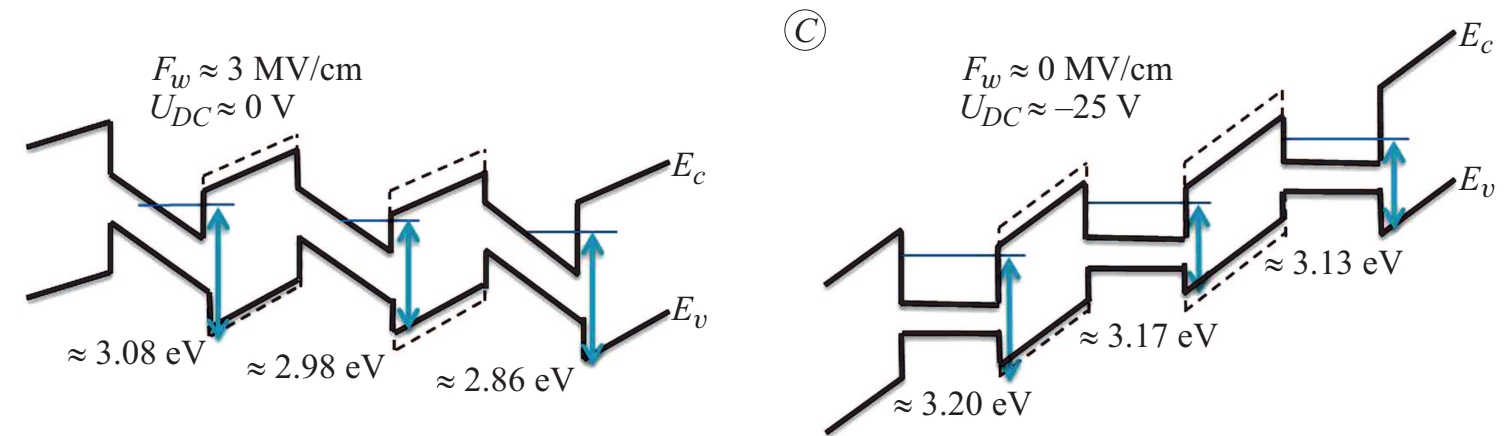

(D)

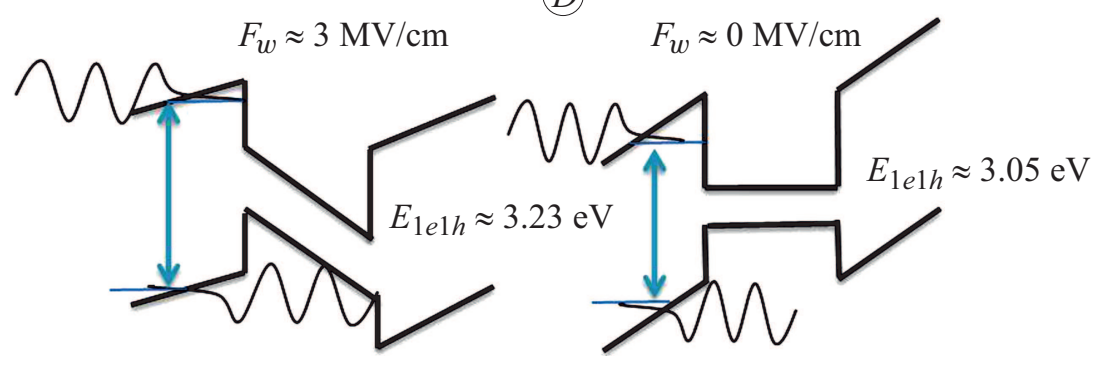

Рис. 4. Энергетические диаграммы различных типов межзонных переходов, наблюдаемых в спектрах ЭП исследованных образцов.

спектре от известного значения ширины запрещенной зоны для объемного $\mathrm{GaN}$ и связано с проявлением эффекта Франца-Келдыша. Другой причиной может быть встраивание индия из КЯ в слои $p-\mathrm{GaN}$ и $n-\mathrm{GaN}$, что также приводит к уменьшению соответствующей ширины запрещенной зоны.

Сдвиги линий $A-D$ при изменении обратного напряжения на $p-n$-переходе связаны с эффектом Штарка. Так как в данной работе исследуется влияние внутренних пьезоэлектрических полей на КЯ, то рассмотрим подробнее поведение линии $A$. Используя положения спектральных линий $A$ для всех образцов, были рассчитаны соответствующие модули напряженности пьезоэлектрических полей с помощью численного решения уравнения Шредингера с гамильтонианом

$$
H=H_{0}+e F_{w} z
$$

где $H_{0}$ - гамильтониан системы с КЯ с конечными стенками, $e-$ заряд электрона, $z-$ координата оси, направленной вдоль [0001], $F_{\mathrm{ext}}$ - напряженность элек- трического поля в КЯ [24]. В расчетах были использованы эффективные массы электронов $m_{e}$ и дырок $m_{h}$ из [25], $m_{0}-$ масса электрона. В результате были получены энергии связанных состояний для электронов и дырок. Используя выражение для ширины запрещенной зоны $E_{g}=0.675 x+3.44(1-x)-1.31(1-x) x[26,27]$ и отношение энергетического разрыва валентной зоны и зоны проводимости на гетерогранице $\mathrm{InGaN} / \mathrm{GaN}$ $\Delta E_{c}: \Delta E_{v}=70: 30$ [28], можно определять энергию перехода между невозбужденными состояниями носителей. Напряженность внутреннего электрического поля $F_{\text {int }}$ в КЯ являлась подгоночным параметром в формуле для напряженности полного электрического поля в КЯ $F_{w}[11]$ :

$$
F_{w}=-\frac{U_{b i}-U_{D C}-E_{\mathrm{int}} N_{w} L_{w}}{N_{w} L_{w}+N_{b} L_{b}+\frac{L_{d}}{2}}+F_{\mathrm{int}},
$$

где $U_{b i}=\left(k_{\mathrm{B}} T / e\right) \ln \left(N_{a} N_{d} / n_{l}^{2}\right)-$ контактная разность потенциалов, $n_{i}=1.8 \cdot 10^{-10} \mathrm{~cm}^{-3}-$ концентрация носителей собственного $\mathrm{GaN}$ [29], $N_{w}, N_{b}=N_{w}+1-$ 


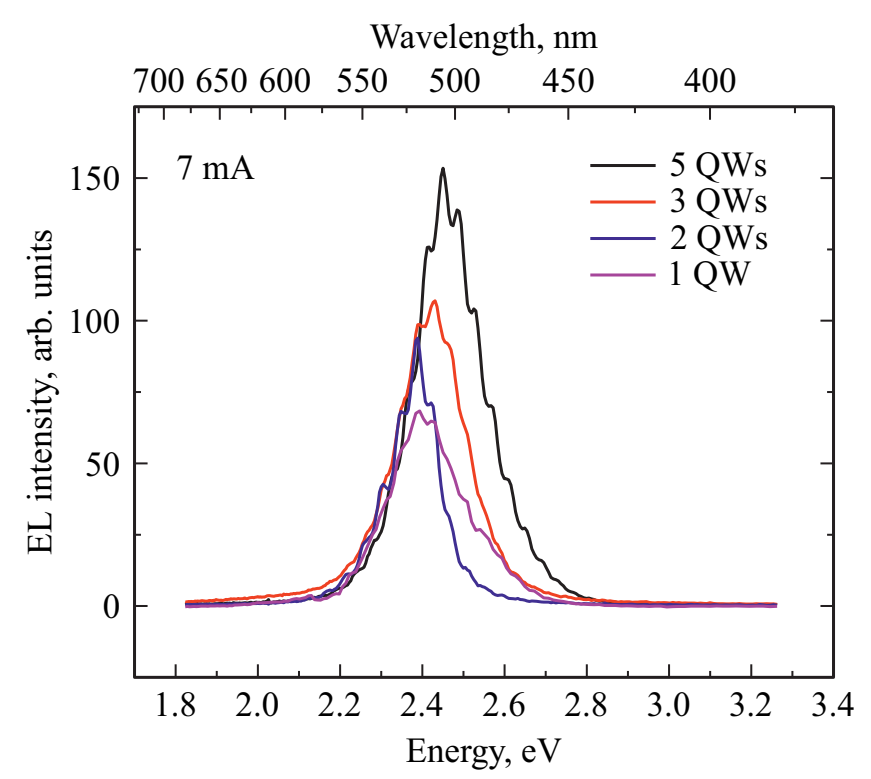

Рис. 5. Спектры ЭЛ (EL) образцов с разным числом КЯ (QW) в активной области при токе 7 мА.

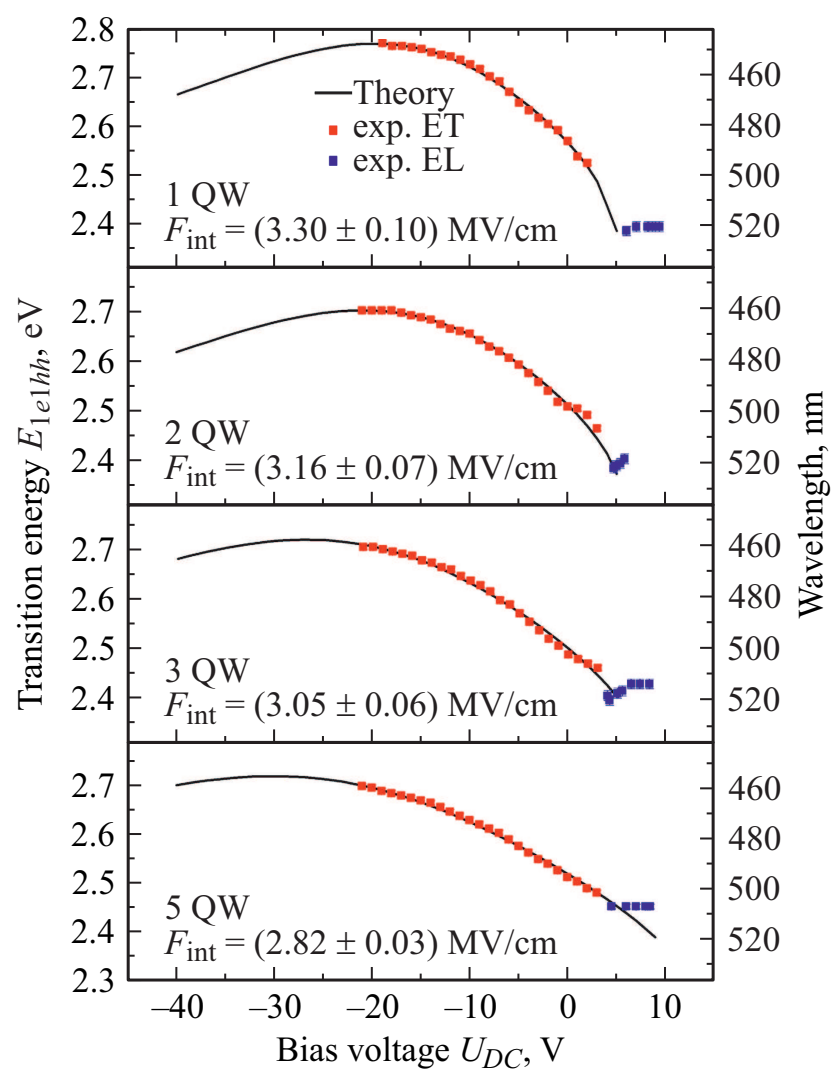

Рис. 6. Зависимость энергии перехода между невозбужденными уровнями в КЯ $(\mathrm{QW})$ зоны проводимости и валентной зоны $E_{1 e 1 h h}$ от смещения $p-n$-перехода $U_{D C}$ для образцов с разным количеством КЯ. Красные и синие квадраты экспериментальные данные, полученные из спектров ЭП (ЕT) и ЭЛ (EL) соответственно. Черная линия — аппроксимация в соответствии с формулами (1)-(3). Для каждого образца приведены напряженности внутреннего электрического поля $F_{\text {int }}$. количество КЯ и барьеров соответственно, $L_{w}, L_{b}-$ ширины КЯ и барьера соответственно. $L_{d}$ - ширина обедненной области рассчитывалась по формуле [30]

$$
\begin{aligned}
L_{d}= & -\left(L_{w} N_{w}+L_{b} W_{b}\right) \\
& +\sqrt{\frac{2 \varepsilon_{0} \varepsilon}{e} \frac{N_{A}+N_{D}}{N_{A} N_{D}}\left(U_{b i}-U_{D C}\right)+\left(L_{w} N_{w}+L_{b} N_{b}\right)^{2}},
\end{aligned}
$$

где $\varepsilon=10.4$ - диэлектрическая проницаемость в слое $\mathrm{GaN}$ [31], $\varepsilon_{0}$ - электрическая постоянная. Результаты аппроксимации приведены на рис. 6. С ростом количества КЯ в активной области, напряженность внутреннего электрического поля уменьшается. Полученные значения электрических полей согласуются с расчетами, сделанными из первых принципов в работе [32].

Поскольку внутренние электрические поля в активной области неоднородны, то увеличение числа КЯ снижает среднюю напряженность электрического поля по всей активной области. Это может быть связано с тем, что слои, ближайшие к $n-\mathrm{GaN}$, выступают в роли буферных для последующих слоев по направлению роста структуры. Таким образом, гетерограница между $n$-GaN и первой КЯ оказывается наиболее деформированной, и напряженность электрического поля в ней достигает $3.30 \mathrm{MB} / \mathrm{cm}$. Следует отметить, что значения напряженностей пьезоэлектрических полей являются эффективными, поскольку были рассчитаны в приближении однородного электрического поля. Поэтому минимальная напряженность электрического поля в структуре с пятью КЯ оказывается ниже, чем $2.82 \mathrm{MB} / \mathrm{cm}$. Такое снижение напряженности в КЯ активной области приводит к сдвигу линии люминесценции и является одним из факторов, повышающих интенсивность люминесценции с увеличением количества КЯ (рис. 5).

\section{4. Заключение}

При комнатной температуре исследованы встроенные электрические поля светодиодных гетероструктур на основе $\mathrm{InGaN} / \mathrm{GaN}$ с различным количеством КЯ методом спектроскопии ЭП. Разработана методика определения энергий межзонных барьерных переходов в слоях $\mathrm{GaN}$, а также переходов между уровнями в квантовых ямах в активной области в слоях InGaN. Определены энергии межзонных переходов, связанных с размытием гетерограниц $\mathrm{InGaN} / \mathrm{GaN}$ вследствие встраивания индия из слоев КЯ InGaN в барьерные слои GaN. Обнаружено, что с ростом числа КЯ в активной области растет количество таких переходов, что свидетельствует о различной степени сегрегации атомов индия в разных КЯ.

Полученные методом ЭП зависимости энергии перехода между невозбужденными уровнями в КЯ от смещения $p-n$-перехода гетероструктуры позволяют определять средние напряженности внутренних электрических полей в КЯ активной области. Наблюдаемое уменьшение 
напряженности электрических полей с ростом количества КЯ в активной области может быть связано с уменьшением механических напряжений на гетерограницах в активной области.

\section{Конфликт интересов}

Авторы заявляют, что у них нет конфликта интересов.

\section{Список литературы}

[1] F. Bernardini, V. Fiorentini, D. Vanderbilt. Phys. Rev. B, 56, R10024 (1997).

[2] M.B. Nardelli, K. Rapcewicz, J. Bernholc. Appl. Phys. Lett., 71, 3135 (1997).

[3] F. Bernardini, V. Fiorentini. Appl. Surf. Sci., 166, 23 (2000).

[4] P.J. Stevens, M. Whitehead, G. Parry, K. Woodbridge. IEEE J. Quant. Electron., 24, 2007 (1988).

[5] D. Holec, Y. Zhang, D.V.S. Rao, M.J. Kappers, C. McAleese, C.J. Humphreys. J. Appl. Phys., 104, 123514 (2008).

[6] S.J. Leem, Y.C. Shin, K.C. Kim, E.H. Kim, Y.M. Sung, Y. Moon, S.M. Hwang, T.G. Kim. J. Cryst. Growth, 311, 103 (2008).

[7] В.П. Кладько, А.В. Кучук, Н.В. Сафрюк, В.Ф. Мачулин, А.Е. Беляев, Р.В. Конакова, Б.С. Явич, Б.Я. Бур, Д.Ю. Казанцев. ФТП, 45 (6), 770 (2011).

[8] A. David, M.J. Grundmann, J.F. Kaeding, N.F. Gardner, T.G. Mihopoulos, M.R. Krames. Appl. Phys. Lett., 92, 053502 (2008).

[9] T.-S.S. Kim, B.-J.J. Ahn, Y. Dong, K.-N.N. Park, J.-G.J.H.H.J.G. Lee, Y. Moon, H.-K.K. Yuh, S.-C.C. Choi, J.-G.J.H.H.J.G. Lee, S.-K.K. Hong, J.H. Song, others. Appl. Phys. Lett., 100, 71910 (2012).

[10] А.Э. Асланян, Л.П. Авакянц, П.Ю. Боков, А.В. Червяков. ФТП, 53, 493 (2019).

[11] Y.D. Jho, J.S. Yahng, E. Oh, D.S. Kim. Appl. Phys. Lett., 79, 1130 (2001).

[12] L.P. Avakyants, A.E. Aslanyan, P.Y. Bokov, A.V. Chervyakov, K.Y. Polozhentsev. Solid-State Electron., 130, 45 (2017).

[13] S. Chichibu, T. Azuhata, T. Sota, S. Nakamura. Appl. Phys. Lett., 69, 4188 (1996).

[14] L.P. Avakyants, A.E. Aslanyan, P.Y. Bokov, K.Y. Polozhentsev, A.V. Chervyakov. Semiconductors, 51, 189 (2017).

[15] K. Jezierski, P. Markiewicz, J. Misiewicz, M. Panek, B. Ściana, R. Korbutowicz, M. Tłaczała. J. Appl. Phys., 77, 4139 (1995).

[16] C.Y. Lai, T.M. Hsu, W.-H.H. Chang, K.-U.U. Tseng, C.-M.M. Lee, C.-C.C. Chuo, J.-I.I. Chyi. J. Appl. Phys., 91, 531 (2002).

[17] F. Renner, P. Kiesel, G.H. Döhler, M. Kneissl, C.G. de Walle, N.M. Johnson. Appl. Phys. Lett., 81, 490 (2002).

[18] C.-K. Sun, J.-C. Liang, X.-Y. Yu. Phys. Rev. Lett., 84, 179 (2000).

[19] M. Kuball, E.-S.S. Jeon, Y.-K.K. Song, A.V. Nurmikko, P. Kozodoy, A. Abare, S. Keller, L.A. Coldren, U.K. Mishra, S.P. DenBaars, others, D.A. Steigerwald. Appl. Phys. Lett., 70, 2580 (1997).

[20] J.-S. Park, D.-Y. Lee, S. Hong, J.W. Kim, B.-K. Kim. Proc. SPIE, 6473, 64731R (2007).

[21] R.J. Kaplar, S.R. Kurtz, D.D. Koleske. Phys. Status Solidi C Conf., 2, 2866 (2005).
[22] C. Wetzel, T. Takeuchi, H. Amano, I. Akasaki. J. Appl. Phys., 85, 3786 (1999).

[23] F. Bernardini, V. Fiorentini. Phys. Status Solidi B, 216, 391 (1999).

[24] D. Ahn, S.L. Chuang. Phys. Rev. B, 34, 9034 (1986).

[25] P. Rinke, M. Winkelnkemper, A. Qteish, D. Bimberg, J. Neugebauer, M. Scheffler. Phys. Rev. B, 77, 075202 (2008).

[26] R.R. Pelá, C. Caetano, M. Marques, L.G. Ferreira, J. Furthmüller, L.K. Teles. Appl. Phys. Lett., 98, 151907 (2011).

[27] P. Schley, R. Goldhahn, G. Gobsch, M. Feneberg, K. Thonke, X. Wang, A. Yoshikawa. Phys. Status Solidi B, 246, 1177 (2009).

[28] C.C. Chen, H.W. Chuang, G.C. Chi, C.C. Chuo, J.I. Chyi. Appl. Phys. Lett., 77, 3758 (2000).

[29] V. Bougrov, M.E. Levinshtein, S.L. Rumyantsev, A. Zubrilov. Properties of Advanced Semiconductor Materials GaN, AlN, InN, BN, SiC, SiGe (John Wiley \& Sons, Inc., N. Y., 2001).

[30] I.H. Brown, I.A. Pope, P.M. Smowton, P. Blood, J.D. Thomson, W.W. Chow, D.P. Bour, M. Kneissl. Appl. Phys. Lett., 86, 131108 (2005).

[31] F. Bernardini, V. Fiorentini, D. Vanderbilt. Phys. Rev. Lett., 79, 3958 (1997).

[32] U.M.E. Christmas, A.D. Andreev, D.A. Faux. J. Appl. Phys., 98, 073522 (2005).

Редактор Г.А. Оганесян

\section{Study of the internal electric fields in active area InGaN/GaN-based LED structures with different numbers of quantum wells by electrotransmission spectroscopy}

A.E. Aslanyan ${ }^{1}$, L.P. Avakyants' ${ }^{1}$, A.V. Chervyakov' ${ }^{1}$, A.N. Turkin ${ }^{1}$, S.S. Mirzai ${ }^{1}$, V.A. Kureshov ${ }^{2}$, D.R. Sabitov ${ }^{2}$, A.A. Marmalyuk ${ }^{2}$

${ }^{1}$ M.V. Lomonosov Moscow State University (Faculty of Physics), 119991 Moscow, Russia

${ }^{2}$ Sigm Plus Co,

117342 Moscow, Russia

Abstract The transmission spectroscopy was used to study the internal electric fields of InGaN/GaN LED green heterostructures with a different number of quantum wells in the active region. The frequencies of the observed spectral lines with possible types of interband transitions are assigned. An increase in the number of quantum well - quantum barrier interband transitions was detected with an increase in the number of quantum wells, which is explained by the unequal segregation of In atoms into different $\mathrm{GaN}$ barrier layers. Using a series of electrical transmission spectra at different bias of the $p-n$-junction, the intensity of internal electric fields in quantum wells was calculated. It was found that with an increase in the number of quantum wells, the intensity of the internal piezoelectric field decreases from 3.20 to $2.82 \mathrm{MV} / \mathrm{cm}$. 